\title{
Different forms of malnutrition among under five children in Bangladesh: a cross sectional study on prevalence and determinants
}

\author{
Sunanda Das and Jahida Gulshan*
}

\begin{abstract}
Background: This empirical study investigated the extent of malnutrition and factors associated with malnutrition amid children aged 0-59 months in Bangladesh using Bangladesh Demographic Health Survey data, 2014.

Methods: To examine the extent of malnutrition among the children under five in Bangladesh, we used Height-forage, weight-for-height and weight-for-age. The association between the selected factors and nutritional status were assessed and logistic regression models were fitted for the three indicators.

Results: $36.2 \%$ children are stunted, 15\% are wasted and 33\% are underweight. Prevalence of stunting or underweight is lowest amongst children aged 0-6 months and highest at the age of 18 to 23 months (stunted $48 \%$ and underweight $37 \%$ ). Wasting is highest in $0-6$ months. Odd of being stunted is 30\% to $50 \%$ higher in Sylhet division as compared to other divisions. Other key covariates for stunting are urban area $(\mathrm{OR}=1.226, p$-value $=0.004)$, no or primary education of father ( $\mathrm{OR}=1.318, p$-value $<0.001)$, no or primary education of mother $(\mathrm{OR}=1.22, p$-value $=0.002)$, underweight mothers $(\mathrm{OR}=1.76, p$-value $<0.001)$ and wealth index poorest $(\mathrm{OR}=2.892, p$-value $<0.001)$. Important covariates for wasting are mother's occupation as physical labor $(\mathrm{OR}=1.208, p$-value $=0.018)$, underweight mothers $(\mathrm{OR}=2.145, p$-value $<0.001)$ and wealth index poorest $(\mathrm{OR}=2.892, p$-value $<0.001)$. For underweight main covariates are: no or primary education of father $(\mathrm{OR}=1.182, p$-value $=0.011)$, no or primary education of mother $(\mathrm{OR}=1.214, p$-value $=0.002)$, mothers in physical labor $(\mathrm{OR}=1.289, p$-value $<0.001)$, underweight mothers $(\mathrm{OR}=2.625, p$-value $<0.001)$ and wealth index poorest $(\mathrm{OR}=2$. $315, p$-value $<0.001)$.

Conclusions: In addition to the ongoing programs to improve child health, government may wish to design targeted nutrition intervention strategies to make sure that health information and health education are easily accessible for parents. The most vulnerable groups including the children from poorest socio-economic group or children in the urban area require special attention. Mothers should also be given focus while designing intervention programs.
\end{abstract}

Keywords: Malnutrition, Stunted, Wasted, Underweight, Logistic regression, Odds ratio

\footnotetext{
* Correspondence: gulshan@isrt.ac.bd

Institute of Statistical Research and Training, University of Dhaka, Dhaka,

Bangladesh
} 


\section{Background}

Theoretically malnutrition is a term that refers to both under-nutrition and over-nutrition. People are malnourished if the calories and protein they take through their diet are not sufficient for their growth and maintenance or due to ill health, they are not able to make complete use of the food they eat (under nutrition) or if they consume too many calories (over-nutrition) [1]. In this paper, we considered under-nutrition and malnutrition equivalently.

The physical and/or mental development of children can be hampered by poor nutrition during childhood which consequently may lead to a greater risk of casualty from communicable diseases or additional critical infections which ultimately end in a bigger economic burden of a society [2,3]. Evidently, malnutrition among children and mothers adversely affect the growth of development in both national and international economic arena as well as health and sustainable developments [4]. Malnutrition is the salient source of 3.5 million deaths globally, and responsible for $35 \%$ of the morbidities among children under five [5] which undoubtedly, defines malnutrition as a prime cause for critical health and development disorders faced by people, mostly children in developing countries [6]. Characteristics of children suffering from malnutrition include stunting or chronic malnutrition (low height for age), wasting or acute malnutrition (Low weight-for-height) or being underweight for their age [7].

In the nineties, 50.6 million under-five children were estimated to be malnourished in developing countries and more than $20 \%$ of the extremely malnourished with a critical illness that led to hospitalization were estimated to endure a case-fatality [8]. The prevalence of worldwide stunting and underweight reduced from 34 to $27 \%$ and 27 to $22 \%$ respectively during 1990-2000 [9]. Eastern and South-eastern Asia as well as the Latin America and the Caribbean made extensive improvements in this regard by achieving large declines, while Africa or South-central Asia constantly suffered towering levels of malnutrition. During this period, the numbers of stunted children in Africa stepped up from 40 million to 45 million, and number of and underweight children mounted from 25 million to 31 million [10, 11].

Sixteen percent of the under-five children were estimated to be underweight worldwide in 2011 [8, 11]. As compared to the other parts of the world the prevalence of underweight children in South Asia was visibly towering. Conversely, a very small part of the global occurrence of underweight are accounted for Latin America and the Caribbean and Central and Eastern Europe/ Commonwealth of Independent States (3 and 2\% respectively) [11]. In the same year, $70 \%$ wasted children of the world were found in Asia with a dreadful level of prevalence of wasting (16\%) in South Asia [11].
The steep decline in the prevalence rate of malnutrition starting from the early 1980s including the fall in the proportions of stunted, wasted and underweight during the time periods: 1984-1985 1994-1995, and 20042005 evidently indicates that Bangladesh made significant achievements in fighting against child malnutrition in last few decades, $[8,12]$.

However, researchers and policymakers cannot disregard the fact that the high prevalence of malnutrition is, even now, one of the principal sources of morbidity and mortality among children [13, 14]. The prevalence of stunted children in Bangladesh was $41 \%$ in 2011, 43.2\% in 2007 and 51\% in 2004 [15-17]. Nonetheless, it is still inadequate to attain the target of the malnutrition prevalence of $34 \%$ of the Millennium Development Goal (MDG), 2015 [18].

In 2012, Save the Children reported that $48.6 \%$ of under five children in Bangladesh were stunted; $13.3 \%$ were wasted and $37.4 \%$ were underweight; the number of stunted children in the poorest quintile of the population were two times the same in the richest quintile [14]. Regardless of a noticeable economic growth from the early 1990s, (of 5 to $6 \%$ a year) the health and wellbeing of Bangladeshi children are challenged by their meager nutritional status which in turn delays the growth towards accomplishment of the millennium development goals not only on maternal and child mortality but also on poverty [19].

Malnutrition is a complicated issue which depends on multiple factors and presumably vary over time and thus needs to be studied on a constant basis. The goal of the study is to delineate malnutrition in terms of different indicators of malnutrition, to assess the prevalence of malnutrition, to examine the association of child malnutrition with chosen demographic and socio-economic factors as well as environmental and health-related factors and to determine the contribution of the selected factors to a child being stunted, wasted and underweight in Bangladesh based on the latest demographic and health survey data of Bangladesh.

\section{Methods}

\section{Data and variables}

In this study relevant data were extracted from the Bangladesh Demographic and Health Survey (BDHS), 2014 which was designed to be the source of typical results for the urban and the rural areas of the country as well as for each of the seven administrative divisions and for the country as a whole. One of the specific objectives of the 2014 BDHS was to examine the nutritional status of under five children using anthropometric measurements (weight and height). The National Institute of Population Research and Training (NIPORT) of the Ministry of Health and Family Welfare of Bangladesh 
conducted the survey that employed a nationally representative two-stage stratified sample of households. Out of a total of 17,989 chosen households, interviews were effectively done in $98 \%$ of all the occupied households [4].

\section{Response variables}

The widely used measures for malnutrition, defined by three anthropometric indicators stunted (height-for-age), wasted (weight-for-height) and underweight (weight-forage) [20] are considered as the response variables. Children are categorized into two groups, 'suffering from malnutrition' and 'not suffering from malnutrition', for each of the three indicators following the guidelines in the national report of Bangladesh [4] and the World Health Organization [11, 21].

\section{Covariates/predictor variables}

A set of interrelated demographic, socioeconomic and environmental factors associated with child nutrition is considered which are: Age of child, sex of child (male, female), place of residence (urban, rural), division (Barisal, Chittagong, Dhaka, Khulna, Rajshahi, Rangpur, Sylhet), religion (Muslim and non-Muslim), parent's education (no education or primary, secondary or higher), father's occupation (physical labor related, service/desk job/business, others), mother's occupation (physical labor related, service/desk job/ business, others), wealth index (poorest, poorer, middle, richer, richest), sources of drinking water (safe or unsafe), type of toilet facilities (hygienic and non-hygienic), total number of living children and Birth order number.

\section{Methodology}

Three widely used measures for malnutrition, height-forage, weight-for-height, and weight-for-age, were used to examine the extent of malnutrition among under-five children in the country using the most recent Bangladesh Demographic and Health Survey (BDHS), 2014 data. A two-stage stratified sample of households is used for the survey. Sampling weights are applied in the analysis of the BDHS data to confirm a genuine representation of the findings of the survey at the national and domain levels. The estimation procedure used the weighting factors because the sample was not selfweighted [22]. Sample weights are used in all analyses for proper standard error and p-value estimation to make sample data representative of the entire population.

The anthropometric method for measuring the nutritional status includes three widely used indicators to assess the growth of children: height-for-age, weight-forheight, and weight-for-age. A child is considered stunted if $(\mathrm{s})$ he is, in terms of height-for-age, more than two standard deviations below the median $(-2 \mathrm{SD})$ of the WHO reference population. A child is wasted when (s)he is more than two standard deviations below (-2 SD) the reference median for weight-for-height. A child is classified as underweight if his or her weight-for-age is lower than two standard deviations (-2 SD) from the median of the reference population.

This paper classifies the nutritional status of under five children on the basis of Z-scores, mathematically defined as:

$$
\begin{aligned}
Z \text { score }= & \text { (individual value (height or weight }) \\
& - \text { median value of reference population }) \\
& / \text { (standard deviation value of reference } \\
& \text { population })
\end{aligned}
$$

A Z-score below - 2 in any of these three indices indicates malnutrition.

WHO AnthroPlus Software (version 3.2.2, 2011) has been used to calculate the Z-scores [23].

Bivariate analysis was performed using cross tables and Chi-square tests to investigate the association between the selected factors and nutritional status. The factors for which $p$-values were less than 0.25 in the bivariate analysis were chosen as the covariates in the logistic regression model [24]. To estimate the effect of the factors on nutritional status, three different logistic regression models (considering the dependent variable to be (i) stunting, (ii) wasting and (iii) underweight) were considered.

\section{Results}

According to BDHS 2014, 36.2\% children are stunted, $15 \%$ are wasted and 33\% are underweight in Bangladesh. The prevalence of stunting, wasting and underweight at different levels or categories of the selected factors are examined and results are presented in Table 1. The estimated parameters of the multiple logistic regression models fitted to identify the contribution of the background factors on malnutrition status of the children are shown in Tables 2, 3 and and 4.

The factors significantly associated ( $p$-value $<0.001)$ with stunting are age of child, Place of residence, division, father's education, mother's education, father's occupation, mother's occupation, mother's BMI, wealth index, toilet facility, number of living children and birth order. Age of child, sex of child, Place of residence, division, mother's BMI and wealth index are significantly associated ( $p$-value $<0.05$ ) with wasting. Factors having significant association with underweight are same as the factors associated with stunting.

To determine the contribution of the associated factors on each of the different measures of nutritional status of under-five children in Bangladesh, we fitted logistic regression models. Table 2 shows the estimates 
Table 1 Cross-classification of types of malnutrition by Socio-demographic factors

\begin{tabular}{|c|c|c|c|c|c|c|c|}
\hline \multirow{2}{*}{\multicolumn{2}{|c|}{ Background Characteristics }} & \multicolumn{2}{|l|}{ Stunting } & \multicolumn{2}{|l|}{ Wasting } & \multicolumn{2}{|l|}{ Underweight } \\
\hline & & Stunted & Not Stunted & Wasted & Not Wasted & Under weight & Not Underweight \\
\hline \multirow[t]{10}{*}{ Age (in months) } & $<6$ & $87(14.2 \%)$ & $525(85.8 \%)$ & $138(22.6 \%)$ & $473(77.4 \%)$ & $112(18.3 \%)$ & 499 (81.7\%) \\
\hline & $6-8$ & $65(16.5 \%)$ & $329(83.5 \%)$ & $65(16.5 \%)$ & $329(83.5 \%)$ & $71(18.0 \%)$ & $323(82.0 \%)$ \\
\hline & $9-11$ & $100(22.9 \%)$ & $337(77.1 \%)$ & 89 (20.4\%) & $348(79.6 \%)$ & $110(25.1 \%)$ & $328(74.9 \%)$ \\
\hline & $12-17$ & $262(32.3 \%)$ & 549 (67.7\%) & $146(18.0 \%)$ & 665 (82.0\%) & $230(28.4 \%)$ & $581(71.6 \%)$ \\
\hline & $18-23$ & $357(48.0 \%)$ & $386(52.0 \%)$ & $93(12.5 \%)$ & $650(87.5 \%)$ & $259(34.8 \%)$ & $485(65.2 \%)$ \\
\hline & $24-35$ & $574(40.3 \%)$ & 849 (59.7\%) & $190(13.4 \%)$ & $1232(86.6 \%)$ & $527(37.0 \%)$ & $896(63.0 \%)$ \\
\hline & $36-47$ & $632(45.2 \%)$ & $766(54.8 \%)$ & $164(11.7 \%)$ & $1233(88.3 \%)$ & $521(37.3 \%)$ & $876(62.7 \%)$ \\
\hline & $48-59$ & $537(38.7 \%)$ & $852(61.3 \%)$ & $192(13.8 \%)$ & $1196(86.2 \%)$ & $529(38.1 \%)$ & 859 (61.9\%) \\
\hline & & $\chi^{2}=341.334$ & $d f=7$ & $\chi^{2}=63.748$ & $d f=7$ & $\chi^{2}=159.735$ & $d f=7$ \\
\hline & & \multicolumn{2}{|c|}{$p$-value $=0.000$} & \multicolumn{2}{|c|}{$p$-value $=0.000$} & \multicolumn{2}{|l|}{$p$-value $=0.000$} \\
\hline \multirow[t]{4}{*}{ Sex } & Male & $1374(36.6 \%)$ & $2378(63.4 \%)$ & $593(15.8 \%)$ & $3159(84.2 \%)$ & 1203 (32.1\%) & 2549 (67.9\%) \\
\hline & Female & $1240(35.9 \%)$ & $2214(64.1 \%)$ & 485 (14.0\%) & $2969(86.0 \%)$ & 1156 (33.5\%) & 2298 (66.5\%) \\
\hline & & $\chi^{2}=0.403$ & $d f=1$ & $\chi^{2}=4.395$ & $d f=1$ & $\chi^{2}=1.613$ & $d f=1$ \\
\hline & & \multicolumn{2}{|l|}{$p$-value $=0.271$} & \multicolumn{2}{|c|}{$p$-value $=0.019$} & \multicolumn{2}{|l|}{$p$-value $=0.107$} \\
\hline \multirow[t]{4}{*}{ Place of Residence } & Urban & $554(30.5 \%)$ & $1264(69.5 \%)$ & $238(13.1 \%)$ & $1581(86.9 \%)$ & $483(26.6 \%)$ & $1335(73.4 \%)$ \\
\hline & Rural & $2060(38.2 \%)$ & $3328(61.8 \%)$ & $841(15.6 \%)$ & $4546(84.4 \%)$ & 1876 (34.8\%) & $3512(65.2 \%)$ \\
\hline & & $\chi^{2}=35.411$ & $d f=1$ & $\chi^{2}=6.823$ & $d f=1$ & $\chi^{2}=42.021$ & $d f=1$ \\
\hline & & \multicolumn{2}{|c|}{$p$-value $=0.000$} & \multicolumn{2}{|c|}{$p$-value $=0.005$} & \multicolumn{2}{|l|}{$p$-value $=0.000$} \\
\hline \multirow[t]{9}{*}{ Division } & Barisal & 160 (38.5\%) & $256(61.5 \%)$ & $80(19.2 \%)$ & $337(80.8 \%)$ & $151(36.3 \%)$ & $243(57.0 \%)$ \\
\hline & Chittagong & $588(38.4 \%)$ & $943(61.6 \%)$ & $250(16.3 \%)$ & $1282(83.7 \%)$ & $546(35.6 \%)$ & $1110(62.3 \%)$ \\
\hline & Dhaka & $866(34.2 \%)$ & $1668(65.8 \%)$ & $317(12.5 \%)$ & $2216(87.5 \%)$ & 728 (28.7\%) & $1677(70.6 \%)$ \\
\hline & Khulna & $156(28.3 \%)$ & 395 (71.7\%) & $80(14.5 \%)$ & $471(85.5 \%)$ & 145 (26.3\%) & 457 (62.2\%) \\
\hline & Rajshahi & $226(30.2 \%)$ & $522(69.8 \%)$ & $136(18.2 \%)$ & $611(81.8 \%)$ & $244(32.6 \%)$ & $672(67.2 \%)$ \\
\hline & Rangpur & $273(37.8 \%)$ & $450(62.2 \%)$ & $129(17.8 \%)$ & $595(82.2 \%)$ & $266(36.7 \%)$ & $532(63.9 \%)$ \\
\hline & Sylhet & 345 (49.1\%) & 357 (50.9\%) & 87 (12.4\%) & $616(87.6 \%)$ & 279 (39.7\%) & 369 (62.3\%) \\
\hline & & $\chi^{2}=86.690$ & $d f=6$ & $\chi^{2}=34.564$ & $d f=6$ & $\chi^{2}=57.872$ & $d f=6$ \\
\hline & & \multicolumn{2}{|c|}{$p$-value $=0.000$} & \multicolumn{2}{|c|}{$p$-value $=0.000$} & \multicolumn{2}{|l|}{$p$-value $=0.000$} \\
\hline \multirow[t]{4}{*}{ Religion } & Muslim & $2405(36.5 \%)$ & $4188(63.5 \%)$ & 999 (15.2\%) & $5594(84.8 \%)$ & $2158(32.7 \%)$ & $4435(67.3 \%)$ \\
\hline & Others & 209 (34.0\%) & 405 (66.0\%) & $80(13.0 \%)$ & $534(87.0 \%)$ & $201(32.8 \%)$ & $412(67.2 \%)$ \\
\hline & & $\chi^{2}=1.446$ & $d f=1$ & $\chi^{2}=1.989$ & $d f=1$ & $\chi^{2}=.001$ & $d f=1$ \\
\hline & & \multicolumn{2}{|l|}{$p$-value $=0.123$} & \multicolumn{2}{|c|}{$p$-value $=0.087$} & \multicolumn{2}{|l|}{$p$-value $=0.505$} \\
\hline \multirow[t]{4}{*}{ Father's Education } & Primary or below & $1763(43.8 \%)$ & $2265(56.2 \%)$ & $628(15.6 \%)$ & $3400(84.4 \%)$ & $1558(38.7 \%)$ & $2470(61.3 \%)$ \\
\hline & Secondary or Higher & 851 (26.8\%) & $2327(73.2 \%)$ & $451(14.2 \%)$ & $2727(85.8 \%)$ & 801 (25.2\%) & 2377 (74.8\%) \\
\hline & & $\chi^{2}=221.848$ & $d f=1$ & $\chi^{2}=2.733$ & $d f=1$ & $\chi^{2}=146.479$ & $d f=1$ \\
\hline & & $p$-value $=0.00$ & & $p$-value $=0.0$ & & $p$-value $=0.000$ & \\
\hline Mother's Education & Primary or below & $1437(45.0 \%)$ & $1759(55.0 \%)$ & $498(15.6 \%)$ & 2699 (84.4\%) & 1279 (40.0\%) & $1918(60.0 \%)$ \\
\hline & Secondary or Higher & $1177(29.4 \%)$ & $2833(70.6 \%)$ & $581(14.5 \%)$ & $3429(85.5 \%)$ & 1080 (26.9\%) & $2930(73.1 \%)$ \\
\hline & & $\chi^{2}=187.495$ & $d f=1$ & $\chi^{2}=1.655$ & $d f=1$ & $\chi^{2}=138.083$ & $d f=1$ \\
\hline & & $p$-value $=0.00$ & & $p$-value $=0.1$ & & $p$-value $=0.000$ & \\
\hline
\end{tabular}


Table 1 Cross-classification of types of malnutrition by Socio-demographic factors (Continued)

\begin{tabular}{|c|c|c|c|c|c|c|c|}
\hline \multirow[t]{5}{*}{ Father's Occupation } & Physical labor & $1970(39.4 \%)$ & $3030(60.6 \%)$ & 767 (15.3\%) & $4233(84.7 \%)$ & 1733 (34.7\%) & $3267(65.3 \%)$ \\
\hline & Service/business & $585(29.2 \%)$ & $1418(70.8 \%)$ & $289(14.4 \%)$ & $1715(85.6 \%)$ & $568(28.3 \%)$ & $1436(71.7 \%)$ \\
\hline & Others & 59 (29.2\%) & $143(70.8 \%)$ & $23(11.3 \%)$ & $180(88.7 \%)$ & $58(28.7 \%)$ & $144(71.3 \%)$ \\
\hline & & $\chi^{2}=68.780$ & $d f=2$ & $\chi^{2}=3.125$ & $d f=2$ & $\chi^{2}=27.451$ & $d f=2$ \\
\hline & & \multicolumn{2}{|c|}{$p$-value $=0.000$} & \multicolumn{2}{|c|}{$p$-value $=0.210$} & \multicolumn{2}{|l|}{$p$-value $=0.000$} \\
\hline \multirow[t]{5}{*}{ Mother's Occupation } & Physical labor & $725(42.0 \%)$ & $1002(58.0 \%)$ & $288(16.7 \%)$ & $1439(83.3 \%)$ & $690(39.9 \%)$ & $1038(60.1 \%)$ \\
\hline & Service/business & 85 (30.1\%) & 197 (69.9\%) & $43(15.2 \%)$ & $240(84.8 \%)$ & 79 (27.9\%) & 204 (72.1\%) \\
\hline & Others & $1804(34.7 \%)$ & $3392(65.3 \%)$ & $748(14.4 \%)$ & $4448(85.6 \%)$ & 1591 (30.6\%) & $3606(69.4 \%)$ \\
\hline & & $\chi^{2}=34.347$ & $d f=2$ & $\chi^{2}=5.307$ & $d f=2$ & $\chi^{2}=54.232$ & $d f=2$ \\
\hline & & \multicolumn{2}{|l|}{$p$-value $=0.000$} & \multicolumn{2}{|c|}{$p$-value $=0.070$} & \multicolumn{2}{|l|}{$p$-value $=0.000$} \\
\hline \multirow{5}{*}{$\begin{array}{l}\text { Mother's BMl } \\
\text { (weight/height }^{2} \text { ) }\end{array}$} & Under weight & $691 Z(43.0 \%)$ & 916 (57.0\%) & 310 (19.3\%) & $1297(80.7 \%)$ & 722 (44.9\%) & $885(55.1 \%)$ \\
\hline & Normal weight & $1622(37.9 \%)$ & $2658(62.1 \%)$ & 645 (15.1\%) & $3635(84.9 \%)$ & $1380(32.2 \%)$ & $2901(67.8 \%)$ \\
\hline & Overweight/obese & $301(22.8 \%)$ & $1018(77.2 \%)$ & $123(9.3 \%)$ & $1196(90.7 \%)$ & $257(19.5 \%)$ & $1062(80.5 \%)$ \\
\hline & & $\chi^{2}=139.600$ & $d f=2$ & $\chi^{2}=56.650$ & $d f=2$ & $\chi^{2}=214.179$ & $d f=2$ \\
\hline & & \multicolumn{2}{|c|}{$p$-value $=0.000$} & \multicolumn{2}{|c|}{$p$-value $=0.000$} & \multicolumn{2}{|l|}{$p$-value $=0.000$} \\
\hline \multirow[t]{7}{*}{ Wealth Index } & Poorest & $811(49.6 \%)$ & $824(50.4 \%)$ & $286(17.5 \%)$ & $1561(85.6 \%)$ & $731(44.7 \%)$ & 905 (55.3\%) \\
\hline & Poorer & $573(42.2 \%)$ & $785(57.8 \%)$ & $233(17.2 \%)$ & $1304(83.0 \%)$ & $526(38.7 \%)$ & 832 (61.3\%) \\
\hline & Middle & $523(36.7 \%)$ & $904(63.3 \%)$ & $190(13.3 \%)$ & $1249(82.6 \%)$ & $463(32.5 \%)$ & $963(67.5 \%)$ \\
\hline & Richer & 447 (31.1\%) & 989 (68.9\%) & $198(13.8 \%)$ & $1249(83.6 \%)$ & 395 (27.5\%) & $1041(72.5 \%)$ \\
\hline & Richest & 259 (19.2\%) & $1091(80.8 \%)$ & $172(12.7 \%)$ & $1145(85.3 \%)$ & $244(18.1 \%)$ & $1106(81.9 \%)$ \\
\hline & & $\chi^{2}=333.376$ & $d f=4$ & $\chi^{2}=23.090$ & $d f=4$ & $\chi^{2}=277.887$ & $d f=4$ \\
\hline & & \multicolumn{2}{|l|}{$p$-value $=0.000$} & \multicolumn{2}{|c|}{$p$-value $=0.000$} & \multicolumn{2}{|l|}{$p$-value $=0.000$} \\
\hline \multirow{4}{*}{$\begin{array}{l}\text { Source of Drinking } \\
\text { Water }\end{array}$} & Unsafe & $278(35.5 \%)$ & $504(64.5 \%)$ & $120(15.4 \%)$ & $661(84.6 \%)$ & $253(32.4 \%)$ & $528(67.6 \%)$ \\
\hline & Safe & $2336(36.4 \%)$ & $4089(63.6 \%)$ & 959 (14.9\%) & $5466(85.1 \%)$ & 2106 (32.8\%) & $4319(67.2 \%)$ \\
\hline & & $\chi^{2}=0.197$ & $d f=1$ & $\chi^{2}=0.105$ & $d f=1$ & $\chi^{2}=0.047$ & $d f=1$ \\
\hline & & \multicolumn{2}{|l|}{$p$-value $=0.344$} & \multicolumn{2}{|c|}{$p$-value $=0.390$} & \multicolumn{2}{|l|}{$p$-value $=0.432$} \\
\hline \multirow[t]{4}{*}{ Toilet Facility } & Hygienic & $2167(35.4 \%)$ & $3949(64.6 \%)$ & $906(14.8 \%)$ & $5210(85.2 \%)$ & 1955 (32.0\%) & $4160(68.0 \%)$ \\
\hline & Unhygienic & $448(41.1 \%)$ & $643(58.9 \%)$ & $173(15.9 \%)$ & $918(84.1 \%)$ & 404 (37.0\%) & $687(63.0 \%)$ \\
\hline & & $\chi^{2}=12.701$ & $d f=1$ & $\chi^{2}=0.792$ & $d f=1$ & $\chi^{2}=10.764$ & $d f=1$ \\
\hline & & \multicolumn{2}{|l|}{$p$-value $=0.000$} & \multicolumn{2}{|c|}{$p$-value $=0.199$} & \multicolumn{2}{|l|}{$p$-value $=0.001$} \\
\hline \multirow[t]{4}{*}{ No. of Living Children } & $1-2$ & $1655(33.4 \%)$ & $3307(66.6 \%)$ & $756(15.2 \%)$ & $4205(84.8 \%)$ & 1507 (30.4\%) & $3455(69.6 \%)$ \\
\hline & $3-11$ & $960(42.8 \%)$ & $1285(57.2 \%)$ & 323 (14.4\%) & $1922(85.6 \%)$ & 852 (38.0\%) & $1393(62.0 \%)$ \\
\hline & & $\chi^{2}=59.179$ & $d f=1$ & $\chi^{2}=0.880$ & $d f=1$ & $\chi^{2}=40.336$ & $d f=1$ \\
\hline & & \multicolumn{2}{|l|}{$p$-value $=0.000$} & $p$-value $=0.1$ & & $p$-value $=0.000$ & \\
\hline Birth Order & $1-2$ & $1659(33.6 \%)$ & $3273(66.4 \%)$ & 740 (15.0\%) & $4192(85.0 \%)$ & 1507 (30.6\%) & $3425(69.4 \%)$ \\
\hline & 3 or more & 955 (42.0\%) & $1320(58.0 \%)$ & 339 (14.9\%) & 1935 (85.1\%) & 852 (37.5\%) & $1422(62.5 \%)$ \\
\hline & & $\chi^{2}=46.854$ & $d f=1$ & $\chi^{2}=0.011$ & $d f=1$ & $\chi^{2}=33.763$ & $d f=1$ \\
\hline & & $p$-value $=0.00$ & & $p$-value $=0.4$ & & $p$-value $=0.000$ & \\
\hline
\end{tabular}


Table 2 Estimates of parameters of the logistic regression model to determine factors of stunting

\begin{tabular}{|c|c|c|c|c|c|c|c|}
\hline Background characteristics & & $\beta$ & S.E.(ß) & Wald statistics & $\mathrm{df}$ & Sig. & Odds ratio \\
\hline \multirow[t]{8}{*}{ Age groups (in months) } & $<6$ & -1.406 & .133 & 111.049 & 1 & .000 & .245 \\
\hline & $6-8$ & -1.198 & .151 & 62.954 & 1 & .000 & .302 \\
\hline & $9-11$ & -.766 & .131 & 33.925 & 1 & .000 & .465 \\
\hline & $12-17$ & -.279 & .098 & 8.018 & 1 & .005 & .757 \\
\hline & $18-23$ & .455 & .097 & 21.895 & 1 & .000 & 1.576 \\
\hline & $24-35$ & .134 & .081 & 2.721 & 1 & .099 & 1.143 \\
\hline & $36-47$ & .347 & .081 & 18.323 & 1 & .000 & 1.414 \\
\hline & $48-59(r)$ & & & & & & \\
\hline \multirow[t]{2}{*}{ Place of residence } & Urban & .204 & .072 & 8.134 & 1 & .004 & 1.226 \\
\hline & Rural (r) & & & & & & \\
\hline \multirow[t]{7}{*}{ Division } & Barisal & -.388 & .136 & 8.191 & 1 & .004 & .678 \\
\hline & Chittagong & -.183 & .100 & 3.340 & 1 & .068 & .833 \\
\hline & Dhaka & -.448 & .095 & 22.441 & 1 & .000 & .639 \\
\hline & Khulna & -.736 & .131 & 31.647 & 1 & .000 & .479 \\
\hline & Rajshahi & -.837 & .119 & 49.134 & 1 & .000 & .433 \\
\hline & Rangpur & -.442 & .118 & 14.148 & 1 & .000 & .643 \\
\hline & Sylhet (r) & & & & & & \\
\hline \multirow[t]{2}{*}{ Religion } & Muslim & .192 & .096 & 3.992 & 1 & .046 & 1.212 \\
\hline & Others ( $r$ ) & & & & & & \\
\hline \multirow[t]{2}{*}{ Father's education } & No or Primary education & .276 & .065 & 18.152 & 1 & .000 & 1.318 \\
\hline & Secondary or Higher (r) & & & & & & \\
\hline \multirow[t]{2}{*}{ Mother's education } & No or Primary education & .200 & .063 & 9.972 & 1 & .002 & 1.222 \\
\hline & Secondary or Higher ( $r$ ) & & & & & & \\
\hline \multirow[t]{3}{*}{ Father's Occupation } & physical labor related & .283 & .168 & 2.827 & 1 & .093 & 1.327 \\
\hline & service/desk job/business & .192 & .173 & 1.223 & 1 & .269 & 1.211 \\
\hline & others (r) & & & & & & \\
\hline \multirow[t]{3}{*}{ Mother's Occupation } & physical labor related & .115 & .062 & 3.396 & 1 & .065 & 1.122 \\
\hline & service/desk job/business & -.076 & .144 & .276 & 1 & .600 & .927 \\
\hline & others ( $r$ ) & & & & & & \\
\hline \multirow[t]{3}{*}{ Mother's BMI (weight/height²) } & Under weight & .566 & .092 & 37.434 & 1 & .000 & 1.761 \\
\hline & Normal weight & .478 & .079 & 36.391 & 1 & .000 & 1.613 \\
\hline & Over weight/Obese (r) & & & & & & \\
\hline \multirow[t]{5}{*}{ Wealth index } & Poorest & 1.062 & .114 & 86.714 & 1 & .000 & 2.892 \\
\hline & Poorer & .910 & .110 & 67.973 & 1 & .000 & 2.484 \\
\hline & Middle & .773 & .103 & 56.275 & 1 & .000 & 2.167 \\
\hline & Richer & .505 & .097 & 26.970 & 1 & .000 & 1.657 \\
\hline & Richest (r) & & & & & & \\
\hline \multirow[t]{2}{*}{ Toilet Facility } & Hygienic & -.142 & .075 & 3.553 & 1 & .059 & .867 \\
\hline & Unhygienic (r) & & & & & & \\
\hline \multirow[t]{2}{*}{ Number of living children } & $1-2$ & .074 & .113 & .430 & 1 & .512 & 1.077 \\
\hline & $3-11(r)$ & & & & & & \\
\hline \multirow[t]{2}{*}{ Birth order } & $1-2$ & -.088 & .112 & .616 & 1 & .433 & .916 \\
\hline & $3-14(r)$ & & & & & & \\
\hline
\end{tabular}


Table 3 Estimates of parameters of the logistic regression model to determine factors affecting malnutrition (wasting)

\begin{tabular}{|c|c|c|c|c|c|c|c|}
\hline Background characteristics & & B & S.E. $(\beta)$ & Wald statistics & $\mathrm{df}$ & Sig. & Odds ratio \\
\hline \multirow[t]{8}{*}{ Age groups (in months) } & $<6$ & .610 & .127 & 22.940 & 1 & .000 & 1.840 \\
\hline & $6-8$ & .192 & .159 & 1.468 & 1 & .226 & 1.212 \\
\hline & $9-11$ & .446 & .145 & 9.512 & 1 & .002 & 1.562 \\
\hline & $12-17$ & .274 & .122 & 5.022 & 1 & .025 & 1.315 \\
\hline & $18-23$ & -.133 & .137 & .932 & 1 & .334 & .876 \\
\hline & $24-35$ & -.050 & .111 & .202 & 1 & .653 & .951 \\
\hline & $36-47$ & -.172 & .115 & 2.242 & 1 & .134 & .842 \\
\hline & $48-59(r)$ & & & & & & \\
\hline \multirow[t]{2}{*}{ Sex } & Male & .124 & .067 & 3.416 & 1 & .065 & 1.133 \\
\hline & Female (r) & & & & & & \\
\hline \multirow[t]{2}{*}{ Place of residence } & Urban & -.069 & .092 & .568 & 1 & .451 & .933 \\
\hline & Rural (r) & & & & & & \\
\hline \multirow[t]{7}{*}{ Division } & Barisal & .539 & .173 & 9.735 & 1 & .002 & 1.714 \\
\hline & Chittagong & .417 & .137 & 9.202 & 1 & .002 & 1.517 \\
\hline & Dhaka & .040 & .133 & .091 & 1 & .763 & 1.041 \\
\hline & Khulna & .257 & .171 & 2.255 & 1 & .133 & 1.294 \\
\hline & Rajshahi & .443 & .154 & 8.288 & 1 & .004 & 1.557 \\
\hline & Rangpur & .415 & .155 & 7.175 & 1 & .007 & 1.514 \\
\hline & Sylhet (r) & & & & & & \\
\hline \multirow[t]{2}{*}{ Religion } & Muslim & .217 & .128 & 2.963 & 1 & .091 & 1.243 \\
\hline & Others ( $r$ ) & & & & & & \\
\hline \multirow[t]{2}{*}{ Mother's Education } & Primary or below & .019 & .079 & .061 & 1 & .805 & 1.020 \\
\hline & Secondary or Higher (r) & & & & & & \\
\hline \multirow[t]{3}{*}{ Father's Occupation } & Physical labor & .264 & .288 & 1.342 & 1 & .247 & 1.303 \\
\hline & Service/business & .342 & .233 & 2.145 & 1 & .143 & 1.408 \\
\hline & Others ( $r$ ) & & & & & & \\
\hline \multirow[t]{3}{*}{ Mother's Occupation } & Physical labor & .189 & .080 & 5.610 & 1 & .018 & 1.208 \\
\hline & Service/business & .105 & .175 & .358 & 1 & .550 & 1.110 \\
\hline & Others (r) & & & & & & \\
\hline \multirow[t]{3}{*}{ Mother's BMI (weight/height ${ }^{2}$ ) } & Under weight & .763 & .123 & 38.815 & 1 & .000 & 2.145 \\
\hline & Normal weight & .487 & .109 & 20.035 & 1 & .000 & 1.627 \\
\hline & Over weight/Obese (r) & & & & & & \\
\hline \multirow[t]{5}{*}{ Wealth index } & Poorest & .083 & .137 & .371 & 1 & .543 & 1.087 \\
\hline & Poorer & .052 & .132 & .159 & 1 & .690 & 1.054 \\
\hline & Middle & -.192 & .127 & 2.298 & 1 & .130 & .825 \\
\hline & Richer & -.060 & .119 & .255 & 1 & .614 & .942 \\
\hline & Richest (r) & & & & & & \\
\hline \multirow[t]{2}{*}{ Toilet Facility } & Hygienic & .050 & .095 & .269 & 1 & .604 & 1.051 \\
\hline & Unhygienic (r) & & & & & & \\
\hline \multirow[t]{2}{*}{ No. of Living Children } & $1-2$ & .048 & .079 & .369 & 1 & .543 & 1.049 \\
\hline & $3-11(r)$ & & & & & & \\
\hline
\end{tabular}

' $\mathrm{r}$ ' represents reference category 
Table 4 Estimates of parameters of the logistic regression model to determine factors affecting malnutrition (underweight)

\begin{tabular}{|c|c|c|c|c|c|c|c|}
\hline Background characteristics & & $\beta$ & S.E.( $\beta)$ & Wald statistics & df & Sig. & $\exp (\beta)$ (odds ratio) \\
\hline \multirow[t]{8}{*}{ Age groups (in months) } & $<6$ & -1.016 & .123 & 68.540 & 1 & .000 & .362 \\
\hline & $6-8$ & -1.068 & .147 & 53.073 & 1 & .000 & .344 \\
\hline & $9-11$ & -.635 & .128 & 24.465 & 1 & .000 & .530 \\
\hline & $12-17$ & -.464 & .100 & 21.458 & 1 & .000 & .629 \\
\hline & $18-23$ & -.146 & .099 & 2.142 & 1 & .143 & .865 \\
\hline & $24-35$ & -.005 & .081 & .004 & 1 & .949 & .995 \\
\hline & $36-47$ & .026 & .081 & .104 & 1 & .748 & 1.027 \\
\hline & $48-59(r)$ & & & & & & \\
\hline \multirow[t]{2}{*}{ Sex } & Male & -.046 & .053 & .745 & 1 & .388 & .956 \\
\hline & Female (r) & & & & & & \\
\hline \multirow[t]{2}{*}{ Place of residence } & Urban & .083 & .072 & 1.344 & 1 & .246 & 1.087 \\
\hline & Rural (r) & & & & & & \\
\hline \multirow[t]{7}{*}{ Division } & Barisal & -.054 & .135 & .158 & 1 & .691 & .948 \\
\hline & Chittagong & .112 & .100 & 1.253 & 1 & .263 & 1.119 \\
\hline & Dhaka & -.286 & .095 & 9.116 & 1 & .003 & .751 \\
\hline & Khulna & -.397 & .131 & 9.131 & 1 & .003 & .672 \\
\hline & Rajshahi & -.273 & .118 & 5.403 & 1 & .020 & .761 \\
\hline & Rangpur & -.056 & .117 & .228 & 1 & .633 & .946 \\
\hline & Sylhet (r) & & & & & & \\
\hline \multirow[t]{2}{*}{ Father's education } & No or Primary education & .167 & .065 & 6.530 & 1 & .011 & 1.182 \\
\hline & Secondary or Higher Education ( $r$ ) & & & & & & \\
\hline \multirow[t]{2}{*}{ Mother's education } & No or Primary education & .194 & .064 & 9.171 & 1 & .002 & 1.214 \\
\hline & Secondary or Higher Education ( $r$ ) & & & & & & \\
\hline \multirow[t]{3}{*}{ Father's Occupation } & physical labor related & .057 & .167 & .118 & 1 & .731 & 1.059 \\
\hline & service/desk job/business & .146 & .172 & .718 & 1 & .397 & 1.157 \\
\hline & others (r) & & & & & & \\
\hline \multirow[t]{3}{*}{ Mother's Occupation } & physical labor related & .254 & .062 & 16.822 & 1 & .000 & 1.289 \\
\hline & service/desk job/business & -.054 & .144 & .140 & 1 & .708 & .947 \\
\hline & others (r) & & & & & & \\
\hline \multirow[t]{3}{*}{ Mother's BMI (weight/height ${ }^{2}$ ) } & Under weight & .965 & .094 & 105.974 & 1 & .000 & 2.625 \\
\hline & Normal weight & .476 & .082 & 33.765 & 1 & .000 & 1.610 \\
\hline & Over weight/Obese (r) & & & & & & \\
\hline \multirow[t]{5}{*}{ Wealth index } & Poorest & .839 & .113 & 55.346 & 1 & .000 & 2.315 \\
\hline & Poorer & .699 & .110 & 40.054 & 1 & .000 & 2.012 \\
\hline & Middle & .548 & .104 & 27.820 & 1 & .000 & 1.729 \\
\hline & Richer & .346 & .099 & 12.291 & 1 & .000 & 1.414 \\
\hline & Richest (r) & & & & & & \\
\hline \multirow[t]{2}{*}{ Number of living children } & $1-2$ & .074 & .114 & .417 & 1 & .519 & 1.076 \\
\hline & $3-11(r)$ & & & & & & \\
\hline \multirow[t]{2}{*}{ Birth order } & $1-2$ & -.096 & .113 & .731 & 1 & .392 & .908 \\
\hline & $3-14(r)$ & & & & & & \\
\hline
\end{tabular}

' $r$ ' represents reference category 
of parameters of the logistic regression model to determine the contribution of factors to stunting of under five years' children along with corresponding p-values and odds ratios.

Odd of being stunted is almost $50 \%$ lower in Khulna $(\mathrm{OR}=0.479, p$-value $<0.001)$ and almost $15 \%$ lower in Chittagong $(\mathrm{OR}=0.833, p$-value $=0.068)$ as compared to Sylhet division. Other key covariates for stunting are urban area $(\mathrm{OR}=1.226, p$-value $=0.004)$, no or primary education of father $(\mathrm{OR}=1.318, p$-value $<0.001)$, no or primary education of mother $(\mathrm{OR}=1.22, p$-value $=$ $0.002)$, underweight mothers $(\mathrm{OR}=1.76, p$-value $<0.001)$ and wealth index poorest $(\mathrm{OR}=2.892, p$-value $<0.001)$.

Table 3 shows the estimates of parameters of the logistic regression model to determine factors of wasted of under five children along with corresponding $p$-values and odds ratios.

Important covariates for wasting are age of children less than six months $(\mathrm{OR}=1.84, p$-value $<0.001), 9-11$ months $(\mathrm{OR}=1.56, p$-value $<0.005)$ and $12-17$ months $(\mathrm{OR}=1.32, \quad p$-value $<0.05)$, division Barisal $((\mathrm{OR}=$ $1.714, p$-value $<0.005)$, division Chittagong $(\mathrm{OR}=1.517$, $p$-value $<0.005)$, division Rajshahi $(\mathrm{OR}=1.557, p$-value $<0.005)$, division Rangpur ( $\mathrm{OR}=1.514, p$-value $<0.01$ ) mother's occupation as physical labor $(\mathrm{OR}=1.208, p$ value $=0.018)$ and underweight mothers $(\mathrm{OR}=2.145, p$ value $<0.001$ ).

Table 4 shows the estimates of parameters of the logistic regression model to determine factors affecting malnutrition (underweight) under five years' children along with corresponding $p$-values and odds ratios.

Main covariates for underweight are age of children less than six months (OR $=0.362$, $p$-value $<0.001), 6-8$ months $(\mathrm{OR}=0.344, p$-value $<0.001), 9-11$ months $(\mathrm{OR}=0.530$, $p$-value $<0.001)$ and $12-17$ months $(\mathrm{OR}=0.629, p$-value $<0.001)$, division Dhaka (OR $=0.751, p$-value $=0.003)$ division Khulna ( $(\mathrm{OR}=0.672$, $p$-value $<0.005)$, division Rajshahi $(\mathrm{OR}=0.761, \quad p$-value $<0.05)$, no or primary education of father $(\mathrm{OR}=1.182, p$-value $=0.011)$, no or primary education of mother $(\mathrm{OR}=1.214, p$-value $=$ $0.002)$, mothers in physical labor $(\mathrm{OR}=1.289, p$-value $<$ $0.001)$, underweight mothers $(\mathrm{OR}=2.625, p$-value $<0.001)$ and wealth index, poorest $(\mathrm{OR}=2.315, p$-value $<0.001)$.

\section{Discussion}

In spite of the outstanding achievements of Bangladesh in consistently reducing the malnutrition rates among children through the last few decades through various intervention programs taken by the Government and the development partners, the findings of this study (with $36 \%$ stunted, 15\% wasted and around 33\% underweight children under age five in the year 2014) state the scope of further improvement of the child nutritional status is Bangladesh.

In this study, age of children, place of residence, division, religion, education of parents, occupation of parents, BMI of mothers, wealth index and toilet facilities used by the household have significant association with child nutritional status.

Malnutrition for stunting and underweight are relatively lower in younger children (less than 18 months). The fraction of stunted or underweight children increases abruptly with the child's age after 18 months and then falls again after 47 months (see Table 1). For both the measures, malnutrition hits the highest point at age 18 to 35 months. In general, wasting decreases as child grows older. Unfortunately, even during the first six months of life, when the majority of the children are breastfed, 14-22\% children remain malnourished as indicated by the three indices. Furthermore, after the first six months, while children are generally given additional foods along with breast milk, the percentage of stunted or underweight children increase gradually with highest amount of children stunted (48\%) and underweight (37\%) at the age of 18 to 23 months, indicating need of attention of the policy makers to the matter. This finding was in agreement with other studies [25-28]. The odds ratios of .245, .302, .465 .757, $1.58,1.14$ and 1.41 respectively for the age groups ( $<6$ months, 6 to 8 months, 9 to 11 months, 12 to 17 months, 18 to 23 months, 24 to 35 months, 36 to 47 months) in the logistic regression model also supports the fact (reference group children aged 48 to 59 months).

Being male gender was identified as a risk factor of malnutrition in several studies [27-31] and our study also found that prevalence of malnutrition in male children is slightly higher as compared to the same in females. However, stunting and underweight were not significantly associated with sex.

In developing countries, the rural-urban disparities in child nutrition outcomes remained persistent since long [32-34] presumably due to the difference in economic levels and poor accessibility to health facilities, education and other factors. Although many of the previous studies identified that rural children are more vulnerable to be malnourished [32-34], this study found that children living in urban area are more vulnerable to be stunted in Bangladesh. The urban population in last few decades has changed a lot due to migration of rural people to urban area for work and other opportunities. Many of these migrated people are poor and cannot afford to nutritional food and health care facilities which might be the underlying reason behind this increased proportion of malnourished children in urban areas and requires attention of the health policy makers into this matter. 
The logistic regression analysis identified significant association between division and malnutrition. Sylhet division is running far behind the other divisions in controlling malnutrition among children in Bangladesh.

The study also indicated that both the parents' education are significantly associated with nutritional status of their children. Children from illiterate father (as well as mother) or father (or mother) with primary education were twenty to thirty percent more likely to remain stunted or underweight in comparison with their counterparts. This finding was coherent with previous studies held in Bangladesh and other countries [35-40]. This is understandable because educated mothers have greater knowledge regardingthe health and nutrition of their children, improved child care, usage of health services, hygiene and sanitation, etc. On the other hand, father's education is also important for the health and nutritional status of his child because of his contribution in household income and his role in decision making forselecting food for the family.

In addition, this study indicated that proportion of malnourished children is higher among children whose mothers are occupied in physical labor related works or others (mainly housewives) compared to the children of mothers in service or business. This may be rationalized as in Bangladesh; people who are occupied in service or business are usually wealthier and are often more educated as well which consequently allows them to know about nutritional foods as well as importance of such food for children.

A mother with good nutritional status is likely to have healthier babies [41], and in our study also, Mother's BMI is found to have negative association with child malnutrition; this reminds us to not to forget the importance of mother's nutritional status while making policies for lowering or reducing child malnutrition. Because good nutritional status is essential for a mother not only for breastfeeding but also for recovery from physical and possibly emotional stress during pregnancy and after labor in order to cope with raising and caring children.

Wealth index is, as expected [36, 38-42]; negatively associated with malnutrition and odd of being malnourished is substantially high among the poorer groups. This undoubtedly points at the unmet need of policies for poorer groups of people in Bangladesh.

Last but not the least, presence of hygienic toilet facilities was found as an important factor that was found associated with child nutritional status. Children from households with hygienic latrine facilities were less likely to be stunted as compared to their counterparts. This finding was also consistent with other studies [9, $13,35]$. This is very rational in the sense that unhygienic toilets are often causes of diarrheal and other diseases, which, as a consequence, may turn children malnourished.

\section{Limitations}

The study has certain limitations. First of all, since this study was based on a cross-sectional data. and as a result, exploring the association between selected factors and prevalence of malnutrition cannot establish causal association between the two. Secondly, due to unavailability of the data on potential confounders including diet, physical exercise, and smoking behavior of the parents, these were not included in analysis. Third, the definition of urban and rural areas in Bangladesh has been changed over time with the most rapid growth in urbanization. As a result, some areas, earlier classified as rural in the previous BDHSs were considered urban in the more recent BDHSs, which may bring in some error in urban-rural calculations. Fourth, Mymensingh is a new division created from the Dhaka division and this information is not available in the 2014 BDHS.

\section{Conclusion}

The nutritional status of under five children is not only a susceptible indicator of the health and nutrition of a country but also can be considered as a measurement of the quality of life as well as a development indicator as it portrays the intensity of development as a whole governed by poverty, low socio-economic status and the prevalence of chronic diseases [29]. Studying malnutrition, on a continuous basis, is essential since it replicates the accumulative outcome of socio-economic, health and nutritional drawbacks and which may vary over time.

The results of this study confirm that there are quiet rooms to perk up the child nutritional status in Bangladesh. To reduce the burden of malnutrition among children, a joint effort by the government, nongovernmental organizations and the community is absolutely necessary in an equitable manner to improve the nutritional status of children. In addition to the ongoing programs to improve child health, Government may wish to design targeted nutrition intervention strategies with better understanding of target group to reduce childhood malnutrition. Additional to the program to confirm the easier access to health information and health education to parents, surveillance and assessment need to be regularly reviewed with special attention to be given to vulnerable groups such as poorest or children in the urban area. A healthy mother can give birth to healthy children, thus for upgrading the nutritional status of children, focus of early intervention programs 
should not only be on children but also on their mothers.

Last but not the least, in addition to the continuing programs and efforts taken by Bangladesh Government and other development partners, the authors would like to recommend that health and nutrition education should also be a fundamental part of the entire education process.

\section{Acknowledgement}

The authors are thankful to the National Institute of Population Research and Training (NIPORT), Bangladesh for providing the BDHS, 2014 data for public usage. Authors are also thankful to the reviewers for their effective and valuable comments.

\section{Funding}

Not applicable.

\section{Availability of data and materials}

The data is available online in a public domain with all identifier information removed and can be accessed from the website http://dhsprogram.com/ data/available-datasets.cfm.

\section{Authors' contributions}

JG had the basic idea for the study and supervised the research work. SD performed the statistical analysis and prepared the first draft including results and tabs. Both JG and SD contributed in the literature review, discussion section and finalized the manuscript. Both the authors read and approved the final manuscript.

\section{Competing interests}

The authors declare that they have no competing interests.

\section{Consent for publication}

Not applicable. This manuscript does not contain any data on any individual (there are no individual details, images or videos).

\section{Ethics approval and consent to participate}

As mentioned earlier, this study is based on analysis of an existing survey data obtained from Bangladesh Demographic and Health Survey, 2014 that was collected by means of a joint effort of NIPORT, ICF International (USA) and Mitra and Associates. This study does not need an ethical approval since it is based on a secondary data available on the respective website for public usage.

Received: 16 February 2016 Accepted: 19 December 2016 Published online: 03 January 2017

\section{References}

1. UNICEF: Malnutrition definition. http://www.unicef.org/progressforchildren/ 2006n4/malnutritiondefinition.html. Accessed 22 May 2016.

2. Chen LC, Chowdhury A, Huffman SL. Anthropometric assessment of energyprotein malnutrition and subsequent risk of mortality among preschool aged children. Am J Clin Nutr. 1980;33(8):1836-45.

3. Bardosono S, Sastroamidjojo SL. Determinants of child malnutrition during the 1999 economic crisis in selected poor areas of Indonesia. Asia Pac J Clin Nutr. 2007;16(3):512-26.

4. National Institute of Population Research and Training - NIPORT/Bangladesh, Mitra and Associates/Bangladesh, and ICF International. Bangladesh Demographic and Health Survey 2014. Dhaka: NIPORT, Mitra and Associates, and ICF International; 2016. https://dhsprogram.com/pubs/pdf/FR311/FR311. pdf. Accessed 8 Apr 2016.

5. Black RE, Allen LH, Bhutta ZA, Caulfield LE, de Onis M, Ezzati M. Maternal and child under-nutrition: global and regional exposures and health consequences. Lancet. 2008;371:243-60.

6. Mason, D.S., Philip, M.S., and Rae, G. Disease Control priorities in developing Countries, 2nd edition, John B. http://csd.wustl.edu/Publications/ Documents/WP09-39.pdf. Accessed 16 Jul 2014.
7. United Nations $4^{\text {th }}$ report-the world nutrition situation: nutrition throughout life cycle. Final report to the ACC/SCN by the Commission on the Nutrition Challenges of the $21^{\text {st }}$ Century. New York, NY: United Nations, 2000.

8. Ahmed T, Ali M, Ullah MM, Choudhury IA, Haque ME, Salam MA. Mortality in severely malnourished children with diarrhoea and use of a standardised management protocol. Lancet. 1999;353:1919-22.

9. Caulifield LE, de Onis M, Blossner M, Black RE. Under-nutrition as an underlying cause of child deaths associated with diarrhoea, pneumonia, malaria, and measles. Am J Clin Nutr. 2004;80:193-8.

10. de Onis M, Blössner M, Borghi E, Morris R, Frongillo EA. Methodology for estimating regional and global trends of child malnutrition. Int J Epidemiol. 2004;33:1260-70.

11. United Nations Children's Fund, World Health Organization, The World Bank UNICEF-WHO-World Bank Joint Child Malnutrition Estimates. 2011 revision (completed July 2012). http://www.who.int/nutgrowthdb/estimates/en/. Accessed 15 Jan 2016.

12. Islam A, Biswas T. Chronic Stunting among under 5 children in Bangladesh a Situation Analysis. Adv Pediatr Res. 2015;2:18. http://dx.doi.org/10.12715/ apr.2015.2.18. Accessed 26 March, 2016.

13. Bairagi R, Chowdhury MK. Socioeconomic and anthropometric status and mortality of young children in rural Bangladesh. Int J Epidemiol. 1994;23: 1179-84.

14. Tackling Child Malnutrition, Save the Children. 2012. Retrieved from http:// www.cmamforum.org/pool/resources/bangladesh-tackling-childmalnutrition-save-2012.pdf. Accessed 1 Apr 2016.

15. National Institute of Population Research and Training - NIPORT/Bangladesh Mitra and Associates/Bangladesh, and ICF International. Bangladesh Demographic and Health Survey 2011. Dhaka: NIPORT, Mitra and Associates, and ICF International; 2013. Available at http://dhsprogram.com/pubs/pdf/ FR265/FR265.pdf.

16. National Institute of Population Research and Training. Bangladesh demographic and health survey, 2007. Dhaka: National Institute of Population Research and Training; 2007. p. 346.

17. Bangladesh Neonatal and Children Health Profile, Department of Maternal, Newborn, Child and Adolescent Health (MCAMHO). http://www.who.int/ maternal_child_adolescent/epidemiology/profiles/neonatal_child/bgd.pdf. Accessed 28 March, 2016

18. Hasan MT, Soares Magalhaes RJ, Williams GM, Mamun AA. Forecasting the progress towards the target of Millennium Development Goal 1C in children under 5 years of age in Bangladesh. Public Health Nutr. 2015; 18(10):1728-36. doi:10.1017/S1368980014003279.

19. Tran M. Poor nutrition stunts growth of nearly half of under-fives in Bangladesh. 2012. http://www.theguardian.com/global-development/2012/ feb/15/bangladesh-child-malnutrition-stunted-growth. Accessed 18 May 2016.

20. Alam N, Wojtyniak B, Rahaman MM. Anthropometric indicators and risk of death. Am J Clin Nutr. 1989;49:884-8. http://www.ncbi.nlm.nih.gov/ pubmed/2718923. Accessed 23 Mar 2016

21. UNICEF, WHO and World Bank Group. Levels and trends in child malnutrition, UNICEF - WHO - World Bank Group joint child malnutrition estimates, Key findings of the 2015 edition; 2015. https://www.unicef.org/ media/files/JME_2015_edition_Sept_2015.pdf. Accessed 12 Apr 2016.

22. DHS Program. Using Datasets for Analysis. http://dhsprogram.com/data/ Using-DataSets-for-Analysis.cfm\#CP_JUMP_14042. Accessed 23 Mar 2016.

23. WHO. Anthro for personal computers, version 3.2.2, 2011: software for assessing growth and development of the world's children. Geneva: WHO; 2011. http://www.who.int/childgrowth/software/en/. Accessed 1 May 2016.

24. Bursac Z, Gauss CH, Williams DK, Hosmer DW. Purposeful selection of variables in logistic regression. Source Code Biol Med. 2008;3:17. doi:10. 1186/1751-0473-3-17.

25. Babatunde RO, Olagunju FI. Prevalence and determinants of malnutrition among under-five children of farming households in Kwara State, Nigeria. J Agric Sci. 2011;3(3):173-81.

26. Sarkar D, Haldar SK. Socioeconomic Determinants of Child Malnutrition in India: Evidence from NFHS-III. 2014. Available at SSRN: http://ssrn.com/ abstract=2420293 or http://dx.doi.org/10.2139/ssrn.2420293.

27. Fakir AMS, Khan MWR. Determinants of malnutrition among urban slum children in Bangladesh. Health Econ Rev. 2015;5:22.2015. doi:10.1186/ s13561-015-0059-1. 
28. Meshram II, Arlappa N, Balakrishna N, Mallikharjuna Rao K, Laxmaiah A, Brahmam GN. Trends in the prevalence of under-nutrition, nutrient and food intake and predictors of under-nutrition among under five year tribal children in India. Asia Pac J Clin Nutr. 2012;21(4):568-76.

29. Demissie S, Worku A. Magnitude and factors associated with malnutrition in children 6-59 months of Age in pastoral community of dollo Ado district, Somali region, Ethiopia. Sci J Public Health. 2013;1:175-83. doi:10.11648/j. sjph.20130104.12.

30. Zhang J, Shi J, Himes JH, Du Y, Yang S, Shi S, Zhang J. Under-nutrition status of children under 5 years in Chinese rural areas - data from the National Rural Children Growth Standard Survey, 2006. Asia Pac J Clin Nutr. 2011;20(4):584-92.

31. Hien NN, Kam S. Nutritional status and the characteristics related to malnutrition in children under five years of age in Nghean, Vietnam. J Prev Med Public Health. 2008:41(4):232-40.

32. Fotso JC. Urban-rural differentials in child malnutrition: trends and socioeconomic correlates in sub-Saharan Africa. Health Place. 2007;13(1):205-23.

33. Van de Poel E, O'Donnell $\mathrm{O}$, Van Doorslaer E. Are urban children really healthier? Evidence from 47 developing countries. Soc Sci Med. 2007;65(10):1986-2003.

34. Srinivasan CS, Zanello G, Shankar B. Rural-urban disparities in child nutrition in Bangladesh and Nepal. BMC Public Health. 2013;13:581. doi:10.1186/14712458-13-581.

35. Nahar B, Ahmed T, Brown K, Hossain MI. Risk factors associated with severe underweight among young children reporting to a diarrhoea treatment facility in Bangladesh. J Health Popul Nutr. 2010;28:476-83.

36. Khor G, Shari Z. Dual form of malnutrition in the same households in Malaysia, A case study among Malay rural households. Asia Pac J Clin Nutr. 2003;2:427-37.

37. Jesmin A, Yamamoto SS, Malik AA, Haque A. Prevalence and determinants of chronic malnutrition among preschool children: a cross-sectional study in Dhaka City, Bangladesh. J Health Popul Nutr. 2011;29:494-9.

38. Janevic T, Petrovic O, Bjelic I, Kubera A. Risk factors for childhood malnutrition in Roma settlements in Serbia. BMC Public Health. 2010;10:1-8.

39. Ayana AB, Hailemariam TW, Melke AS. Determinants of acute malnutrition among children aged 6-59 months in Public Hospitals, Oromia region, West Ethiopia: a case-control study. BMC Nutrition. 2015;1:34. doi:10.1186/s40795015-0031-9.

40. Urke HB, Bull T, Mittelmark MB. Socioeconomic status and chronic child malnutrition: wealth and maternal education matter more in the Peruvian Andes than nationally. Nutr Res. 2011;31(10):741-7. doi:10.1016/j.nutres.2011. 09.007.

41. Khan REA, Raza MA. Nutritional Status of Children in Bangladesh: Measuring Composite Index of Anthropometric Failure (CIAF) and its Determinants. Pakistan J Commerce Soc Sci. 2014;8(1):11-23.

42. Hong R, Banta JE, Betancourt JA. Relationship between household wealth inequality and chronic childhood under-nutrition in Bangladesh. Int J Equity Health. 2006;5:15. doi:10.1186/1475-9276-5-15.

\section{Submit your next manuscript to BioMed Central and we will help you at every step:}

- We accept pre-submission inquiries

- Our selector tool helps you to find the most relevant journal

- We provide round the clock customer support

- Convenient online submission

- Thorough peer review

- Inclusion in PubMed and all major indexing services

- Maximum visibility for your research

Submit your manuscript at www.biomedcentral.com/submit 